\title{
Findings to Leptomeningeal Metastases in Lung Cancer: A Case Report
}

\author{
Thiago Rodrigo de Noronha ${ }^{1}$ Ricardo Ambrósio Fock ${ }^{1}$ \\ ${ }^{1}$ Hematology Section, Division of Clinical Laboratory, \\ Department of Pharmacy and Clinical Laboratory, \\ University Hospital, University of São Paulo, São Paulo, Brazil

\begin{abstract}
Address for correspondence Ricardo Ambrosio Fock, PhD, Hematology Section, Division of Clinical Laboratory, University Hospital, University of São Paulo, Avenida Lineu Prestes, 2565, São Paulo, SP 05508-900, Brazil (e-mail: hemato@usp.br).
\end{abstract}

\begin{abstract}
Keywords

- leptomeningeal metastases

- lung cancer

- cerebrospinal fluid

Leptomeningeal metastases (LM) is defined as diffuse or focal infiltration of malignant cells into the meninges that bathe the brain and spinal subarachnoid. All malignant tumors may cause LM; however, there are several tumor entities that are more frequently associated with leptomeningeal spread than others such as lung cancer, breast carcinoma, melanoma, and hematologic neoplasms as well as primary brain tumor. Here, we present a case of LM from a 53-year-old woman with lung tumor and show the importance of cerebrospinal fluid analysis in the diagnosis of LM.
\end{abstract}

\section{Introduction}

The leptomeninges are the membranes lining the brain and spinal cord. The cerebrospinal fluid (CSF) lies in between these membranes. ${ }^{1}$ According to the classic hypothesis, the majority of CSF is produced by the choroid plexus and circulates through the ventricles, cisterns, and subarachnoid space to be absorbed into the blood vessel by the arachnoid $\operatorname{villi}^{2}$ (-Fig. 1A).

Leptomeningeal metastases (LM), also referred to as leptomeningeal carcinomatosis, carcinomatous meningitis, or neoplastic meningitis, is defined as diffuse or focal infiltration of malignant cells into the meninges that bathe the brain and spinal subarachnoid. ${ }^{3}$ LM is found in 1 to $5 \%$ of patients with solid tumors, 5 to $15 \%$ of patients with hematologic neoplasms, and 1 to $2 \%$ of patients with primary brain tumors. ${ }^{4}$ In this way, all malignant tumors may cause LM; however, there are several tumor entities that are more frequently associated with leptomeningeal spread than others such as lung cancer, breast carcinoma, melanoma, and hematologic neoplasms as well as primary brain tumors. ${ }^{5}$

The clinical manifestations of LM result from malignant cells clogging the normal exits for CSF causing a fluid buildup and increased pressure in the brain $(-$ Fig. 1B). This increased intracranial pressure can cause nausea and vomiting, headaches and neck pain, vision changes and difficulty walking, and paresthesia and paresis. ${ }^{1.5}$

published online June 15, 2019
DOI https://doi.org/ 10.1055/s-0039-1692513 ISSN 2454-6798.
Diagnosis of LM is based on three assessments types: clinical, imaging, and CSF analysis. The most useful laboratory test is the CSF analysis that commonly shows increased opening pressure $\left(>200 \mathrm{~mm} \mathrm{H}_{2} \mathrm{O}\right)$, high leukocytes $\left(>4 / \mathrm{mm}^{3}\right)$, high protein $(>50 \mathrm{mg} / \mathrm{dL})$, low glucose $(<60 \mathrm{mg} / \mathrm{dL})$, and the presence of malignant cells. ${ }^{4}$

\section{Case Report}

A 53-year-old woman was admitted at the emergency facility complaining of cephalea, drowsiness, mental confusion, and vomiting. Cranial computed tomography (CT) at admission shows hyperdensity of left temporal lobe without hydrocephalus. Carotid CT angiography has no lesions; however, a mass $(3.1 \times 3.0 \mathrm{~cm})$ in the right upper lobe of the lung was detected $(-$ Fig. 1C), with spiculated, irregular margins (arrow, - Fig. 1D) suggestive of malignant neoplasia. CSF analysis was performed on suspicion of LM or central nervous system infection. Opening pressure was not quantified during puncture, but with output under high pressure. CSF test revealed a total protein of $94.1 \mathrm{mg} / \mathrm{dL}$; glucose of 59 $\mathrm{mg} / \mathrm{dL}$; CSF red blood cells of $17 / \mathrm{mm}^{3}$; and white cell count of $20 / \mathrm{mm}^{3}$ with a positive oncotic cytology with $33 \%$ of largesized cells, small-medium nucleus/cytoplasm relationship, abundant cytoplasm and hyperbasophilic, in association with some binucleated cells ( - Fig. 1E, F).

\section{License terms}

$(\circledast) \Theta \circledast$ 

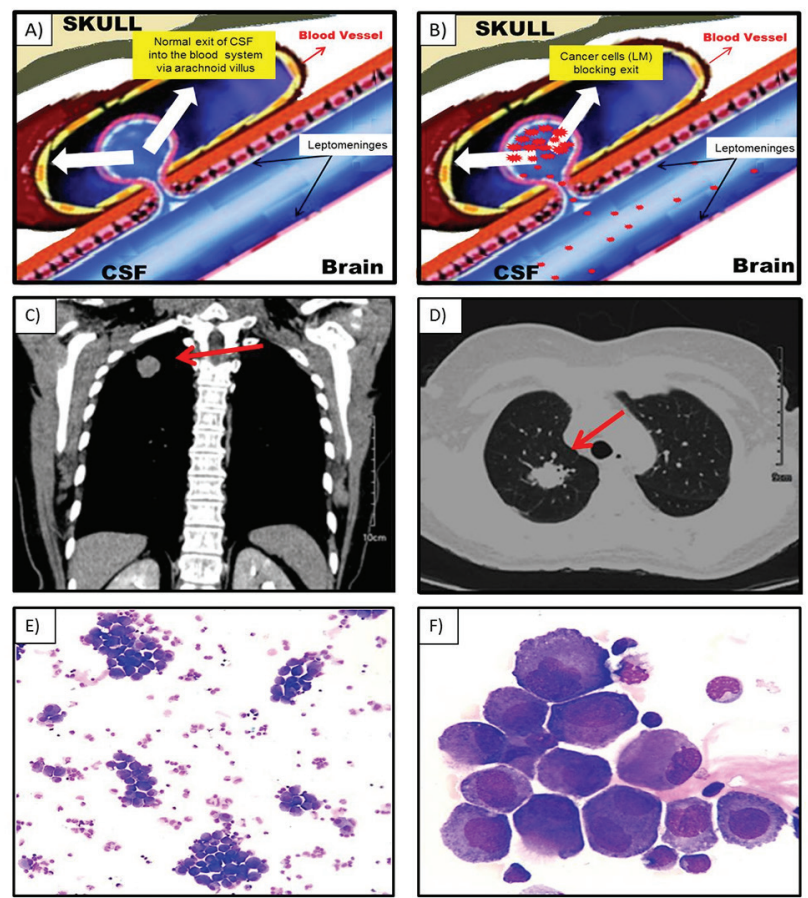

Fig. 1 Normal cerebrospinal fluid flux (A). Cerebrospinal fluid flux blocked by cancer cells $($ B). A mass $(3.1 \times 3.0 \mathrm{~cm})$ in the right upper lobe of the lung and $(\mathbf{C})$ with spiculated, irregular margins (D). Largesized cells, small-medium nucleus/cytoplasm relationship, abundant cytoplasm and hyperbasophilic, in association with some binucleated cells (May-Grünwald stain), (E) with magnification 100× and (F) magnification $1,000 \times$.

\section{Discussion}

Clinical suspicion and early diagnosis are essential to preserve neurological functions and quality of life. The CFS analysis obtained by lumbar puncture is the main complementary test used in the diagnosis of LM. The presence of malignant cells in the CSF is considered the gold standard method for the diagnosis with rare false-positive findings. However, $45 \%$ of patients have a normal initial CFS examination, but the yield is increased to $80 \%$ with analysis of a second CFS puncture. ${ }^{4}$

Other laboratory tests might be helpful, as a flow cytometry and genomic analysis of malignant cells from the CSF. This approach, however, has not yet reached clinical routine. ${ }^{5}$
The prognosis of LM is poor, and survival time of untreated patients is approximately 4 to 6 weeks and treated patients with a median survival duration of 2 to 3 months. ${ }^{4}$

LM remains incurable, so treatments are essentially palliative. The optimal treatment of LM remains poor and is based on two general approaches: to reduce pressure on the brain caused by CSF buildup and to reduce the number of malignant cells (radiotherapy-intrathecal-systemic chemotherapy). If the CSF pressure is elevated, it can be reduced via lumbar puncture or use of some medications. If needed, a permanent drain can be used. ${ }^{1,4}$

The response to treatment in patients with LM is monitored by CSF cytology and clinical improvement in neurological signs and symptoms. Besides, CSF analysis and biochemical markers, no other CSF parameters predict response. Negative cytology requires confirmation by both ventricular and lumbar CSF cytology. ${ }^{4}$ In conclusion, LM remains a therapeutic challenge, and early diagnosis is essential to preserve neurological functions and quality of life. In addition, there is lack of data for specific tumor treatment and the activity of many drugs that have become available within the past years remains unknown.

\section{Author Contribution}

All the authors contributed equally to patient management and writing of this report.

\section{Conflict of Interest}

None declared.

\section{References}

1 Corbin ZA, Nagpal S. Leptomeningeal metastases. JAMA Oncol 2016;2(6):839

2 Brinker T, Stopa E, Morrison J, Klinge P. A new look at cerebrospinal fluid circulation. Fluids Barriers CNS 2014;11:10

3 Ning M, Chunhua M, Rong J, et al. Diagnostic value of circulating tumor cells in cerebrospinal fluid. Open Med (Wars) 2016;11(1):21-24

4 Chamberlain MC. Neoplastic meningitis. Oncologist 2008; 13(9):967-977

5 Roth P, Weller M. Management of neoplastic meningitis. Linchuang Zhongliuxue Zazhi 2015;4(2):26 